\title{
SCIENTIFIC COMMENTARY
}

\section{Do You Need a Pharmacokinetic Model, and, If So, Which One? ${ }^{1}$}

\author{
John G. Wagner ${ }^{2}$ \\ Received Apr. 22, 1975

\section{INTRODUCTION}

My theses are (a) most of the time we cannot determine which specific model applies to one or more sets of data, but only which class of model applies; (b) when linear pharmacokinetics apply, it is usually not necessary to determine which model applies; and (c) when nonlinear pharmacokinetics apply, it is necessary to obtain a partial or complete model to make accurate predictions.

\section{VARIETY OF SIMPLE PHARMACOKINETIC MODELS AVAILABLE}

As part of the defense of the first thesis, a survey will be made of the simple pharmacokinetic models.

Figure 1 shows the linear mammillary disposition models with from one to three compartments. A disposition model is one representing bolus intravenous administration of the drug. In each case, the concentration in compartment 1 is assumed to represent the whole blood (plasma or serum) concentration of unchanged drug. There is only one "one-compartment open model"; there are three different "two-compartment open models"; there are 13 "three-compartment open models." Within each class, the differences between the models resides in the compartment or compartments from which

\footnotetext{
${ }^{1}$ This paper was presented at the First National Congress on Biopharmaceutics and Pharmacokinetics, Barcelona, Spain, May 12-16, 1975. The paper, translated into Spanish, will be published in the Proceedings of the Congress following a slightly different format.

${ }^{2}$ College of Pharmacy and Upjohn Center for Clinical Pharmacology, The University of Michigan, Ann Arbor, Michigan 48104.
} 
ONE COMParTMEnt OPEn Model

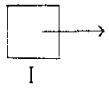

Two Compartment Open Models
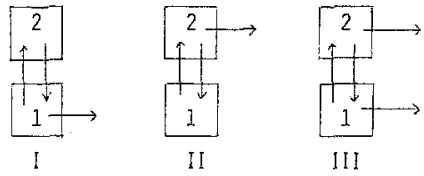

Three Compartment Gpen Models
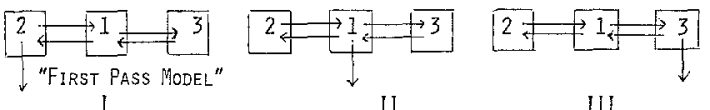

II

III

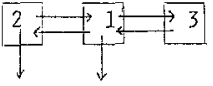

IV

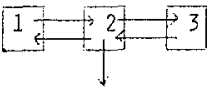

VII

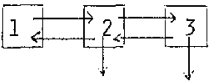

$x$

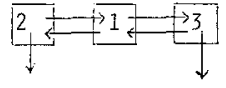

v

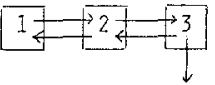

VIII

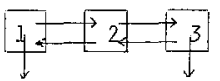

XI

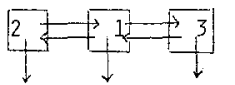

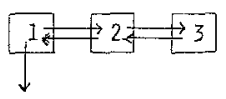

VI

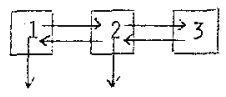

IX

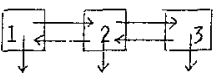

XII

Fig. 1. Linear mammillary disposition models having from one to three compartments. In each case, the concentration in compartment 1 is assumed to represent the measurable concentration of unchanged drug in either whole blood, plasma, or serum.

elimination of drug occurs. In the three-compartment open models, one usually assumes rapid transfer to one of the peripheral compartments and slow transfer or return of drug to and from the other peripheral compartment.

In practice, one decides which class of disposition model applies from the number of exponential terms which are needed to describe the whole blood (plasma or serum) concentration-time curve either following bolus intravenous injection or after a constant-rate intravenous infusion. If only one exponential term is involved, we assign the one-compartment open model. If two exponential terms are involved, we assign the two-compartment open model. If three exponential terms are involved, we assign the threecompartment open model. However, if we measure only in compartment 1 we cannot decide which of the three two-compartment open models or which 
of the 13 three-compartment open models actually applies to a given set of data.

Also, Benet (1) has shown that the number of solvable rate constants, $R$, is given by equation 1 , where $n$ is the number of driving-force compartments.

$$
R=2 n-1
$$

A driving-force compartment is one having one or more exit rate constants. Hence, for the two-compartment open models, $n=2$ and $R=3$. Thus, if we assume either model I or II (of the two-compartment open models shown in Fig. 1), we can estimate the three rate constants-usually designated $k_{12}$, $k_{21}$, and $k_{\mathrm{el}}$. However, if we assume model III (of the two-compartment open models shown in Fig. 1), we cannot estimate the four rate constants if measurements are made only in compartment 1.

For the three-compartment open models, $n=3$ and $R=5$. Hence, if we assume that any one of models I, II, III, VI, VII, and VIII applies, we can estimate the five rate constants. However, if we assume that any of the other seven three-compartment open models applies, we can only estimate five of the total of six or seven rate constants. The real problem, however, is that we must assume a particular model; hence the rate constants estimated are peculiar to our arbitrary choice.

Table I summarizes the linear mammillary disposition models. The class, number of driving-force compartments, maximum number of microscopic rate constants, number of solvable rate parameters, and number of possible models are listed.

A similar set of mammillary disposition models may be formed by replacing first-order elimination by Michaelis-Menten elimination kinetics (2). Hence one would have to determine a $V_{m}$ and a $K_{m}$ value instead of a first-order elimination rate constant. These nonlinear disposition models are summarized in Table II.

DiSanto and Wagner (3) introduced nonlinear tissue-binding models. The general scheme is shown in Fig. 2. It is assumed that there is (are) one or

Table I. Linear Mammillary Disposition Models

\begin{tabular}{lcccc}
\hline Class & $\begin{array}{c}\text { Number of driving- } \\
\text { force compartments }\end{array}$ & $\begin{array}{c}\text { Maximum number } \\
\text { of rate constants }\end{array}$ & $\begin{array}{c}\text { Number of solvable } \\
\text { rate parameters }(2 n-1)\end{array}$ & $\begin{array}{c}\text { Number of } \\
\text { models }\end{array}$ \\
\hline One & 1 & 1 & 1 & 1 \\
Two & 2 & 4 & 3 & 3 \\
Three & 3 & 7 & 5 & 13 \\
& & & & Total $\overline{17}$ \\
\hline
\end{tabular}

${ }^{a} \mathrm{~A}$ driving-force compartment is one with one or more exit rate constants. $n=$ the number of driving-force compartments. 
Table II. Nonlinear Mammillary Disposition Models

\begin{tabular}{lccc}
\hline Class & $\begin{array}{c}\text { Number of driving- } \\
\text { force compartments }\end{array}$ & $\begin{array}{c}\text { Maximum number } \\
\text { of parameters }\end{array}$ & $\begin{array}{c}\text { Number of } \\
\text { possible models }\end{array}$ \\
\hline One & 1 & 2 & 1 \\
Two & 2 & 6 & 3 \\
Three & 3 & 10 & 13 \\
& & & Total $\overline{17}$ \\
\hline
\end{tabular}

${ }^{a}$ Includes all microscopic first-order rate constants and $V_{m}$ and $K_{m}$ values.

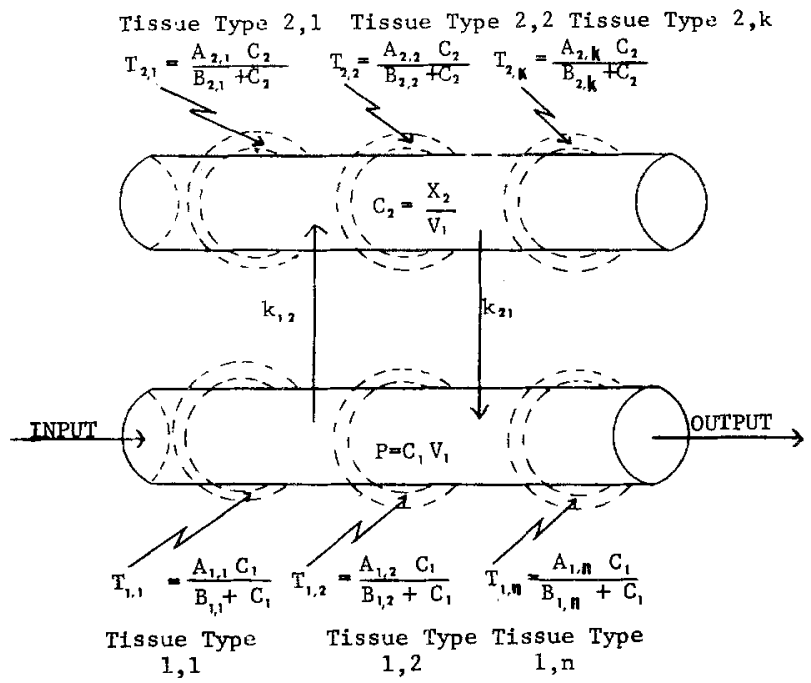

Fig. 2. General scheme for a nonlinear multicompartment model (3) assuming that the drug distributes to one or more homogeneous or heterogeneous compartments representing different structural regions of the system. The drug is introduced into the fluid or aqueous phase of compartment 1 by any input process. This first compartment may be homogeneous (single aqueous phase) or heterogeneous (aqueous phase perfusing one or more tissues of types $1,1,1,2, \ldots, 1, n)$. The drug in solution in the aqueous phase of compartment 1 may also be distributed to the fluid or aqueous phase of compartment $2 . k_{12}$ represents the fraction of drug in the aqueous phase of compartment 1 being transferred per unit time to the aqueous phase of compartment 2, while $k_{21}$ represents the reverse transfer. The second compartment may be homogeneous (single aqueous phase) or heterogeneous (aqueous phase perfusing one or more tissues of types $2,1,2,2, \ldots, 2, k$ ). In each case, the drugtissue "reaction" may be one or more of chemical combination, binding, complexation, adsorption of drug on surface of tissue cells, etc., since the theory does not delineate the mechanism of uptake and release of drug by any given tissue. 
two fluid compartments, designated by the tubelike structures. The relationship between the tissue concentration and the free (non-protein-bound) drug in the fluid is assumed to obey a Langmuir-type equation in each case. We may have one type of fluid and only one type of tissue associated with the fluid, one fiuid and two types of tissue associated with the fluid, etc. We may have two fluids and various combinations of types of tissues associated with the fluids.

Table III summarizes the possible models of this class if it is assumed that there may be either one or two fluids and a maximum number of three types of tissue associated with each fluid. There are 15 possible models if elimination from the inner fluid is assumed to be first order, and another 15 models if elimination is assumed to obey the kinetics of Michaelis and Menten.

Up to this time, we have been considering only intravenous administration. Suppose now that we consider oral or intramuscular administration. If input to the central compartment is represented by a single first-order rate constant, then for each of the disposition models we obtain one more model. Since there are 17 linear mammillary disposition models and 17 nonlinear disposition models, we obtain 34 more models. The 30 tissue-binding models give 30 more models. We may also have the drug going in both directions across the gastrointestinal barrier (i.e., input to the central compartment would be $\stackrel{k_{1}}{\rightleftharpoons}$ instead of $\stackrel{k_{a}}{\rightarrow}$ ); this concept produces a further large number of additional models. We cannot distinguish between these two types of

Table III. Nonlinear Tissue-Binding Models

\begin{tabular}{|c|c|c|c|c|}
\hline \multirow{2}{*}{$\begin{array}{l}\text { Number of fluid } \\
\text { compartments }\end{array}$} & \multirow{2}{*}{$\begin{array}{l}\text { Number of tissues } \\
\text { with fluid No. } 1\end{array}$} & \multirow{2}{*}{$\begin{array}{l}\text { Number of tissues } \\
\text { with fluid No. } 2\end{array}$} & \multicolumn{2}{|c|}{ Number of possible models } \\
\hline & & & First order $^{a} 1$ & Michaelis-Menten $^{\alpha}$ \\
\hline 1 & 1 & 0 & 1 & 1 \\
\hline 1 & 2 & 0 & 1 & 1 \\
\hline 1 & 3 & 0 & 1 & 1 \\
\hline 2 & 1 & 0 & 1 & 1 \\
\hline 2 & 2 & 0 & 1 & 1 \\
\hline 2 & 3 & 0 & 1 & 1 \\
\hline 2 & 1 & 1 & 1 & 1 \\
\hline 2 & 1 & 2 & 1 & 1 \\
\hline 2 & 1 & 3 & 1 & 1 \\
\hline 2 & 2 & 1 & 1 & 1 \\
\hline 2 & 2 & 2 & 1 & 1 \\
\hline 2 & 2 & 3 & 1 & 1 \\
\hline 2 & 3 & 1 & 1 & 1 \\
\hline 2 & 3 & 2 & 1 & 1 \\
\hline \multirow[t]{3}{*}{2} & 3 & 3 & 1 & 1 \\
\hline & & & $\overline{5}$ & $\overline{-5}$ \\
\hline & & \multicolumn{2}{|c|}{ Totals 15} & 15 \\
\hline
\end{tabular}

"Refers to type of elimination kinetics from fluid No. 1. 
input by measuring only in blood. If input (or absorption) is represented by two compartments, then there are eleven possibilities, and nine of them are shown at the bottom of Fig. 3 .

If blood levels following oral administration are fitted by a four-term polyexponential equation, and blood levels following intravenous administration are fitted by a two-term polyexponential equation, then there are 27 possible models to explain the data, as shown in Fig. 3. The disposition portion of the model may be any of the three models shown at the top, and the input portion of the model may be any one of the nine partial models shown at the bottom. It is assumed that the arrowheads on the right-hand side of each of the partial input models at the bottom of Fig. 3 connect with compartment 1 of the disposition models at the top of Fig. 3. In the example cited, it is very difficult, if not impossible, to determine which of the possible 27 models applies to a particular set of data.

Another difficulty is the "pooling effect." In the tissue-binding models, it is extremely difficult to distinguish plasma protein binding from binding of drug to tissue, or binding of drug to one type of tissue from binding to

The Disposition Portion of the Model Could Be:
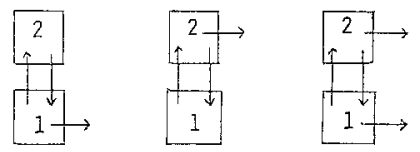

The Input Portion of the Model CoUlod Be:
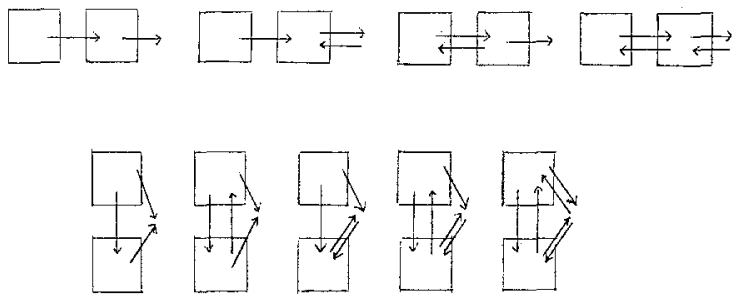

Hence, the Total Mumber of Possible Models $=3 \times 9=27$

Fig. 3. Possible input and disposition portions of models which could describe blood levels following oral administration of a drug where the plasma concentration-time curve gives a four-term polyexponential equation and where blood levels following intravenous administration give only a two-term polyexponential equation. 
another type of tissue. This is because, in a certain parameter space, a sum of two Langmuir equations acts like a single "pooled Langmuir equation." Similarly, Sedman and Wagner (4) showed that parallel Michaelis-Menten paths could be "pooled." Also, frequently, parallel Michaelis-Menten and first-order paths may be "pooled" into one Michaelis-Menten path.

Table IV summarizes 760 possible models that have been surveyed. In addition, there are a large number of flow rate limited models, such as those of Bischoff et al. (5); a typical example is shown in Fig. 4. Figure 4 is an example of a flow rate limited model elaborated by Bischoff et al. (5) to explain distribution and excretion of methotrexate in several mammalian species.

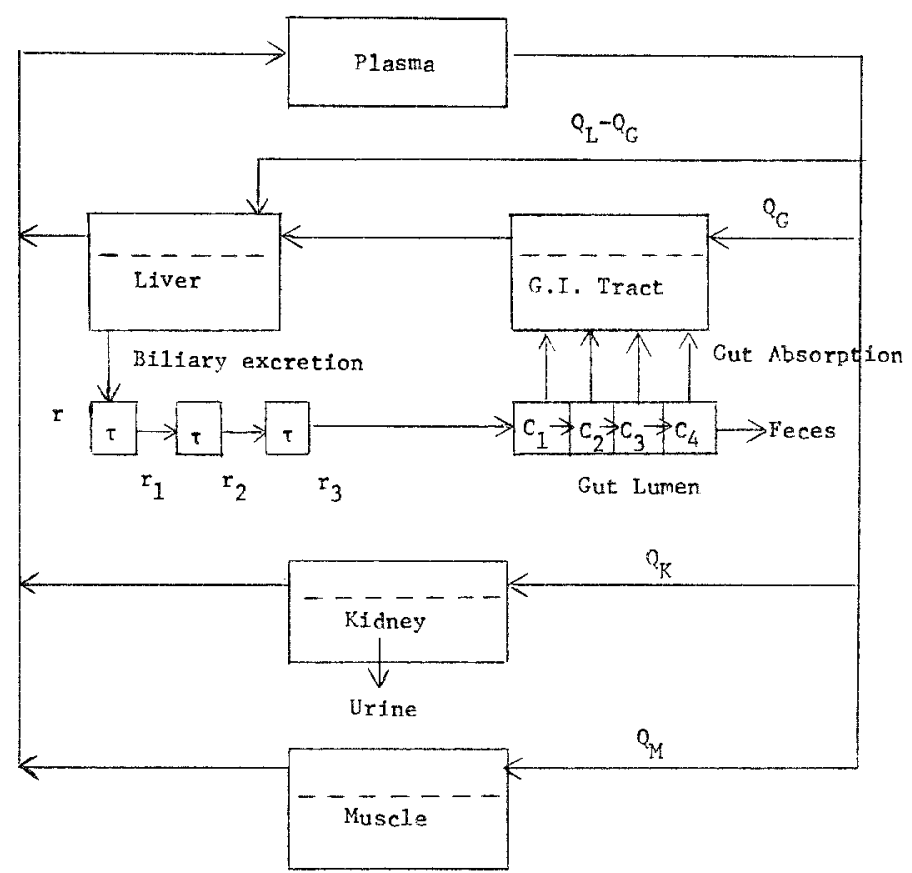

Fig. 4. Blood flow rate limited model posed by Bischoff to explain the distribution and excretion of methotrexate in several animal species. Symbols: $Q$, plasma flow rate to specific organs; $r$, drug transport rate in bile; $\tau$, nominal resident time in bile subcompartments: $C$; drug concentrations in gut lumen subcompartments. Dashed lines within compartments indicate the possibility of tissue-to-plasma distribution within that compartment. 
d. Measurement of the unchanged drug and the major metabolite in urine collections obtained at the same time as experiments (a) and (b) are carried out.

\section{WHICH MODEL?}

Often it does not matter which specific model of a class of models is chosen. For example, the Loo-Riegelman absorption method (6) assumes that model I of the three possible two-compartment open models (see Fig. 1) applies. Recently, Wagner (7) has shown that application of the method will give the correct asymptote and the correct kinetics of absorption in cases where the actual model is any one of the three "three-compartment open models."

The Wagner-Nelson absorption method (8) is based on the one-compartment open model. Recently, Wagner (9) has shown that if the WagnerNelson method is applied to data obeying the two-compartment open model with first-order absorption the Wagner-Nelson data may be resolved to give estimates of both $\alpha$ and $k_{a}$.

The one-compartment open model gives good predictions of average steady-state amounts of drug in the body whenever $\left[\left(\beta / k_{\mathrm{e} 1}\right)\left(1+k_{12} / k_{21}\right] \rightarrow 1\right.$, even though blood levels are best described by the two-compartment open model (10).

Minimum steady-state blood levels are often well predicted by a simple monoexponential equation. This does not mean that the one-compartment open model applies, but rather that at $\tau \mathrm{hr}$ after a dose at steady state all exponential terms, except one, are essentially equal to zero.

\section{WHEN YOU DO NOT NEED A PHARMACOKINETIC MODEL}

My second thesis was that when pharmacokinetics are linear it is usually not necessary to determine which specific model applies. I will now defend this thesis by showing how to perform many of the needed calculations without a model in the classical sense.

\section{Bioavaillability}

First-Pass Effect

The first-pass effect may be assessed using equations 2 and 3:

$$
\begin{gathered}
\theta=\frac{(\text { dose })_{\text {i.v. }} \int_{0}^{\infty} C_{\text {oral }} d t}{(\text { dose })_{\text {oral }} \int_{0}^{\infty} C_{\text {i.v. }} d t} \\
\theta=1-\frac{f_{m}(\text { dose }) \text { i.v. }}{\dot{V}_{\mathrm{b} .1 .} \lambda \int_{0}^{\infty}\left(C_{\text {plasma }}\right) \text { i.v. } d t}
\end{gathered}
$$


In these equations, $C$ refers to the whole blood (plasma or serum) concentration at time $t$, the integrals represent the total area under the concentration-time curve, $f_{m}$ represents the fraction metabolized, $\lambda$ represents the whole blood concentration/plasma concentration ratio, and $\dot{V}_{\text {b.l. }}$ represents the liver blood flow rate. These equations were presented by Rowland (11) and were applied to diphenhydramine by Albert et al. (12). In equations 2 and $3, \theta$ represents the fraction of the oral dose which reaches the general circulation intact. The integrals may be estimated using equations 4 and 5 :

$$
\begin{gathered}
\int_{0}^{\infty} C d t=\int_{0}^{T} C d t+C_{T} / \beta \\
\int_{0}^{\infty} C d t=\int_{0}^{\infty} \sum_{i=1}^{n} A_{i} e^{-E_{i} t} d t=\sum_{i=1}^{n} A_{i} / E_{i}
\end{gathered}
$$

The integral on the right-hand side of equation 4 may be estimated by the trapezoidal rule directly from the $C, t$ data; here, $T$ represents the last sampling time of blood in the study. The area from the last sampling time to infinity is estimated by the term on the far right of equation 4 ; here $C_{T}$ is the concentration at time $T$ and $\beta$ is the first-order elimination rate constant estimated from the very terminal $C, t$ data. To use equation 5 , one would fit the $C, t$ data to a polyexponential equation using a suitable program and a digital computer and then integrate the resulting equation to obtain the appropriate area.

\section{Efficiency of Absorption}

The efficiency of absorption of a drug from a solid oral dosage relative to a solution of the drug may be assessed using equation 6 .

$$
F=\frac{(\text { dose })_{A} \int_{0}^{\infty} C_{B} d t}{(\text { dose })_{B} \int_{0}^{\infty} C_{A} d t}
$$

Here $A$ refers to the reference (or solution) and $B$ refers to the test solid oral dosage form. If $A$ represents the intravenous route of administration, then the $F$ represents the actual fraction of the oral dose which is absorbed (assuming there is no "first-pass" effect). Use of equation 6 involves the assumption that the plasma clearance remains constant. Wagner (13) has reported on an alternative equation in which the area is multiplied by the $\beta$ value obtained for the same set of data.

Rate of Absorption

Rate of absorption may be assessed without a model for linear systems using the deconvolution method introduced by Recigno and Segre (14) and 
studied extensively by Benet and Chiang (15). The general equations for the deconvolution method so as to obtain absorption plots and absorption rate constants directly from blood level data are given below for an orally administered drug:

$$
\begin{aligned}
& H(t)=\int_{0}^{t} F(\theta), G(t-\theta), d \theta=F(t)^{*} G(t) \\
& G(n)=\left\{H(n)-\sum_{i=2}^{n} F(i), G(n-i+1)\right\} / F(1)
\end{aligned}
$$

where $H(n)$ is a function describing the blood concentration-time curve following oral administration, $F(n)$ is a function describing the blood concentration-time curve following bolus intravenous administration, and $G(n)$ is the transfer function describing absorption.

From the general equation 8 , the equations applicable to calculation of individual values of $G_{i}$ are shown below:

$$
\begin{aligned}
& G_{1}=H_{1} / F_{1} \\
& G_{2}=\left(H_{2}-F_{2} G_{1}\right) / F_{1} \\
& G_{3}=\left(H_{3}-F_{2} G_{2}-F_{3} G_{1}\right) / F_{1} \\
& G_{4}=\left(H_{4}-F_{2} G_{3}-F_{3} G_{2}-F_{4} G_{1}\right) / F_{1} \\
& G_{5}=\left(H_{5}-F_{2} G_{4}-F_{3} G_{3}-F_{4} G_{2}-F_{5} G_{1}\right) / F_{1}
\end{aligned}
$$

Benet and Chiang (15) recommend use of the point-area (P-A) method rather than the point-point (P-P) or the area-area (A-A) methods. In the point-area (P-A) method, $H_{i}$ is equal to the blood (plasma or serum) concentration at time $t_{i}$ after oral administration and $F_{i}$ is equal to the area under the bolus intravenous blood (plasma or serum) concentration-time curve between $t_{i}$ and $t_{i+1}$. To apply the deconvolution method illustrated here, the blood (plasma or serum) concentrations must be measured at equally spaced time intervals during the absorption phase, but not at other times. The concentrations have to be measured at the same times following both oral and intravenous administration for the time interval that drug is being absorbed after oral administration. The accuracy of the method depends on how close together the samples are taken during the absorption phase. The same situation holds with the Loo-Riegelman method $(6,7)$. Wagner (7) has shown that greater accuracy in application of the Loo-Riegelman method may be obtained by use of interpolated points as well as the observed data points; the interpolated points were obtained by spline and Akima methods as reported by Fried and Zeitz (16). The same interpolation method may be 
used before application of the deconvolution method. This interpolation method is part of the program AUTOAN (17), which will be described briefly later.

The deconvolution method is illustrated with simulated data in Table V. Equation 14 was used to generate the simulated oral data shown in the fourth column of Table V. Equations $15 \mathrm{a}$ and $15 \mathrm{~b}$ were used to generate the simulated intravenous data and to calculate the area under the curve up to time $t_{i}$, respectively.

$$
\begin{gathered}
C^{\text {p.o. }}=120\left[e^{-0.1 / 425 t}-e^{-1.0455 t}\right] \\
C^{\text {i.v. }}=100 e^{-0.17425 t} \\
\int_{0}^{t_{i}} C^{\text {i.v. }} d t=\frac{100}{0.17425}\left(1-e^{-0.17425 t_{i}}\right)=573.888\left(1-e^{-0.17425 t_{i}}\right)
\end{gathered}
$$

\begin{tabular}{|c|c|c|c|c|c|}
\hline$t_{\mathrm{i}}$ & $\int_{0}^{v_{1}} C^{\mathrm{i} \cdot v \cdot} d t$ & $F_{i}$ & $H_{i}=C^{\mathrm{p} . \mathrm{o}}$ & & $G_{i}$ \\
\hline 0 & $0-$ & 1.77 & 0 & & \\
\hline 1 & 91.77 & & 58.63 & \multicolumn{2}{|c|}{$G_{1}=58.63 / 91.77=0.6389$} \\
\hline 2 & $168.87^{\prime}$ & & 69.86 & \multicolumn{2}{|c|}{$G_{2}=[69.86-(77.10)(0.6389)] / 91.77=0.2245$} \\
\hline 3 & 233.63 & & 65.93 & \multicolumn{2}{|c|}{$\begin{array}{r}G_{3}=[65.93-(77.10)(0.2245)-(64.76)(0.6389)] / 91.77 \\
=0.07896\end{array}$} \\
\hline 4 & 288.04 & & 57.94 & \multicolumn{2}{|c|}{$\begin{aligned} G_{4}=[57.94-(77.10)(0.07896)-(64.76)(0.2245) \\
-(54.41)(0.6389)] / 91.77=0.0278\end{aligned}$} \\
\hline 5 & 333.75 & & 49.57 & \multicolumn{2}{|c|}{$\begin{array}{l}G_{5}=[49.57-(77.10)(0.0278)-(64.76)(0.07896) \\
\quad-(54.41)(0.2245)-(45.71)(0.6389)] / 91.77=0.00974\end{array}$} \\
\hline & $t_{i-1}$ & $G_{i}$ & \multicolumn{2}{|c|}{$\begin{array}{c}\text { Fraction remaining at } \\
\text { absorption site }=G_{i} / 0.6389\end{array}$} & $\begin{array}{l}\text { Actual fraction } \\
\text { remaining }=e^{-1.0455 t_{i-1}}\end{array}$ \\
\hline & 0 & 0.6389 & & 1.0000 & 1.0000 \\
\hline & 1 & 0.2245 & & 0.3514 & 0.3515 \\
\hline & 2 & 0.07896 & & 0.1236 & 0.1236 \\
\hline & 3 & 0.0278 & & 0.0435 & 0.0434 \\
\hline & 4 & 0.00974 & & 0.01524 & 0.01527 \\
\hline
\end{tabular}

Table V. Illustration of the Deconvolution Method ${ }^{a}$

${ }^{a} \ln G_{i}=-0.4478-1.0456 t_{i-1}(r=-1.000)$ (equation 16$), \hat{k}_{a}=1.0456 \mathrm{hr}^{-1}$ (actual value $=$ $1.0455 \mathrm{hr}^{-1}$ ). 
In this simulation, the oral and intravenous doses were assumed to be equal. The values listed under " $G_{i}$ " in Table V arose by application of equations 9-13 to the numbers shown in the third and fourth columns of the table. At the bottom of Table $\mathrm{V}$ the time and $G_{i}$ values are summarized. Equation 16 , the equation for the least-squares $\ln G_{i}, t_{i-1}$ line

$$
\ln G_{i}=-0.4478-1.0456 t_{i-1} \quad(r=-1.000)
$$

is also shown in the table; the slope of this line is the estimated value of $k_{a}$ (absorption rate constant). With these error-free data, the rate constant is estimated extremely accurately by the method. One can also calculate values of "fraction of drug remaining at absorption site" as shown in the lower portion of Table $\mathrm{V}$; these are compared with the actual values found.

To apply the deconvolution method in those cases where the onecompartment open model applies requires only oral data and not intravenous data. This does not appear to have been stated formerly. An illustration is given in Table VI. Once again, equation 14 was used to generate the simulated oral data shown in the fourth column of Table VI. Here oral data were collected long enough to establish the terminal log-linear line shown opposite the data points in the interval 6-12 hr in Table VI. These data were used to generate a $C^{*}, t$ equation (equation 17a) in place of the equation for intravenous administration of the drug. The $C^{*}, t$ equation is integrated stepwise (equation 17b) to obtain areas and the area in each time interval calculated as shown under " $F$ " in the third column in Table VI.

$$
\begin{gathered}
C^{*}=118.95 e^{-0.17343 t} \\
\int_{0}^{t_{i}} C^{*} d t=\frac{118.95}{0.17343}\left(1-e^{-0.17343 t}\right)=685.87\left(1-e^{-0.17343 t}\right)
\end{gathered}
$$

Equations 9-13 are then applied as before to yield the $G_{i}$ values shown in the fourth column of Table VI. The $G_{i}, t_{i-1}$ values are summarized at the bottom of Table VI; the least-squares $\ln G_{i}, t_{i-1}$ equation (equation 18)

$$
\ln G_{i}=-0.6152-1.0559 t_{i-1} \quad(r=0.999)
$$

is shown below the table; the slope of this line is the estimated value of $k_{a}$. In this case, $k_{a}$ was accurately estimated at an error of only $1 \%$ with these error-free data using only the simulated oral data. 
Table VI. Illustration of Deconvolution Method Applied to Oral Data Only (One-Compartment Open Model) ${ }^{a}$

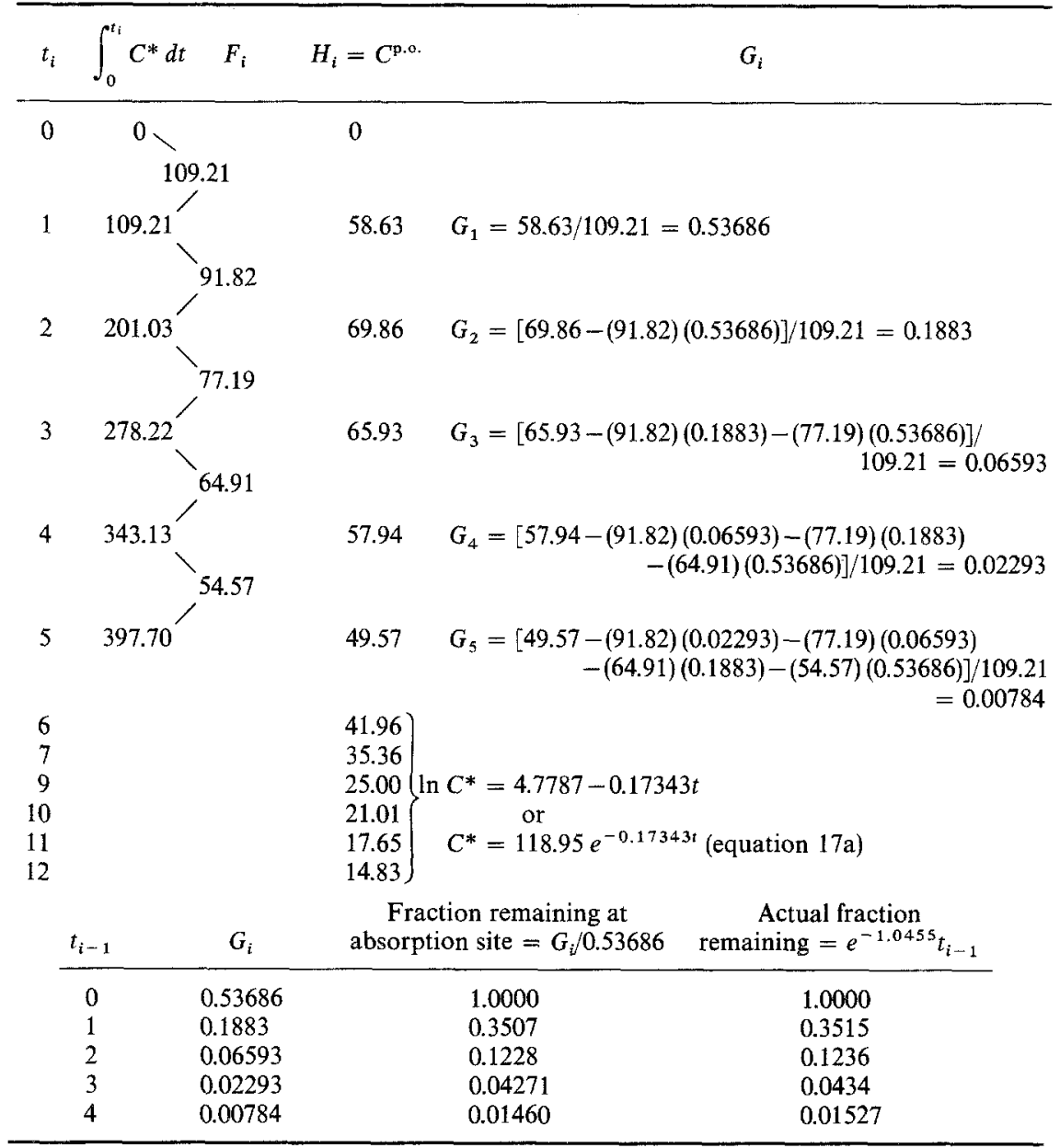

${ }^{a} \ln G_{i}=-0.6152-1.0559 t_{i-1}(r=-0.999)$ (equation 18$), \hat{k}_{a}=1.0559 \mathrm{hr}^{-1}(1 \%$ error) (actual value $=1.0455 \mathrm{hr}^{-1}$ ).

\section{Dosage Regimen Calculations (Linear Systems)}

All dosage regimen calculations may be performed by fitting either single-dose or steady-state blood (plasma or serum) concentration-time data to simple polyexponential equations, and in some cases by use only of the trapezoidal rule to estimate areas. Model parameters do not have to be 
derived from the coefficients and exponents of the polyexponential equations.

$$
C_{1}(t)=\sum_{i=1}^{n} I_{i} e^{-E_{i} t}
$$

Equation 19 is a general equation indicating the polyexponential equation obtained by fitting blood levels observed following a single dose. Here $C_{1}(t)$ represents the blood (plasma or serum) concentration at time $t$ after the dose, $n$ is the number of exponential terms required to explain the data, and $I_{i}$ and $E_{i}$ are the coefficients and exponents of the polyexponential equation. The corresponding steady-state equation is given as equation 20 :

$$
C_{\mathrm{ss}}(t)=\sum_{i=1}^{n}\left\{\frac{I_{i} e^{-E_{i} t}}{1-e^{-E_{i} t}}\right\}
$$

Here, $C_{\text {ss }}(t)$ refers to the steady-state concentration at time $t$ after a dose at steady-state and $\tau$ is the uniform dosing interval. To obtain the time of the maximum concentration at steady state, $t_{\mathrm{ss}}^{\max }$, one differentiates equation 20 and sets the derivative equal to zero as shown by equation 21 :

$$
\frac{d C_{\mathrm{ss}}}{d t}=\sum_{i=1}^{n}\left\{\frac{-E_{i} I_{i} e^{-E_{i} t_{\mathrm{s}} \mathrm{sax}^{\mathrm{ax}}}}{1-e^{-E_{i} \tau}}\right\}=0
$$

If there are only one or two exponential terms (i.e., $n=1$ or 2 ) in the polyexponential equation, then $t_{\mathrm{ss}}^{\max }$ may be obtained from equation 21 by the use of logarithms; however, if there are three or more terms (i.e., $n \geq 3$ ), then one must solve equation 21 for $t_{\mathrm{ss}}^{\text {rama }}$ by an iterative method using an electronic calculator or a digital computer.

Once the value of $t_{\mathrm{ss}}^{\max }$ is known, then the maximum concentration at steady state may be calculated with equation 22 ; the minimum concentration at steady state is given by equation 23 :

$$
\begin{aligned}
C_{\mathrm{ss}}^{\max } & =\sum_{i=1}^{n}\left\{\frac{I_{i} e^{-E_{i} t_{s s}^{\max }}}{1-e^{-E_{i} \tau}}\right\} \\
C_{\mathrm{ss}}^{\min } & =\sum_{i=1}^{n}\left\{\frac{I_{i} e^{-E_{i} \tau}}{1-e^{-E_{i} \tau}}\right\}
\end{aligned}
$$

I would like to propose that the ratio of the loading dose/maintenance may be calculated using equation 24 . This ratio is given by the ratio of the area under the curve during a dosage interval at steady state to the area under the single-dose curve from time 0 to time $\tau$.

$$
\frac{\text { loading dose }}{\text { maintenance dose }}=\frac{\int_{0}^{\tau} C_{\mathrm{Ss}}(t) d t}{\int_{0}^{\tau} C_{1}(t) d t}=\frac{\int_{0}^{\infty} C_{1}(t) d t}{\int_{0}^{\tau} C_{1}(t) d t}=\frac{\sum_{i=1}^{i} I_{i} / E_{i}}{\sum_{i=1}^{n}\left(I_{i} / E_{i}\right)\left(1-e^{-E_{i \tau}}\right)}
$$


The use of the above equation gives a slightly higher value for the ratio than the method used by Krüger-Thiemer (18). A comparison of the two methods is given below for the one-compartment open model with first-order absorption which was always used by Krüger-Thiemer in dosage regimen calculations:

$$
\begin{aligned}
& \frac{\text { loading dose }}{\text { maintenance dose }}=\frac{C_{\mathrm{ss}}^{\min }}{C_{1}^{\min }}=\frac{1}{\left(1-e^{-K \tau}\right)\left(1-e^{-k \tau}\right)} \\
& \frac{\text { loading dose }}{\text { maintenance dose }}=\frac{\bar{C}_{\mathrm{ss}}}{\bar{C}_{1}}=\frac{k-K}{k\left(1-e^{-K \tau}\right)-K\left(1-e^{-k \tau}\right)}
\end{aligned}
$$

Equations 25 and 26 compare the ratio of the loading dose/maintenance dose for the one-compartment open model with first-order absorption shown. Here $k$ is the absorption rate constant and $K$ is the elimination rate constant. The method of Krüger-Thiemer (18) is indicated by equation 25; in this method, the required ratio is equal to the ratio of the minimum concentration at steady state to the minimum concentration at $\tau \mathrm{hr}$ after the first dose. The method I am proposing gives the ratio equal to the ratio of the average concentration at steady state, $\bar{C}_{\mathrm{ss}}$, to the average concentration from 0 to $\tau$ hr after the first dose, $\bar{C}_{1}$. This is indicated by equation 26. For example, if $k=1.0455, \tau=6$, and $K=0.17425$, then equation 25 gives a value of 1.54 and equation 26 gives a value of 1.73 .

If steady-state blood levels are measured at several times after dosing, then one could fit the data to equation 20 rather than using single-dose blood levels to make predictions.

If only minimum steady-state blood levels are measured (i.e., blood levels just before the next dose), such as in the hospital situation, then a model-independent equation is shown as equation 27. This is not useful per se but may be written as either equation 28 or 29 depending on what type of information is available.

$$
C_{\mathrm{ss}}^{\min }=\left[\frac{F}{V_{D}}\right]\left[\frac{\text { fraction remaining at end of dosage interval }}{\text { fraction lost during dosage interval }}\right] D_{M}
$$

In most cases, one can write either

$$
C_{\mathrm{ss}}^{\min }=\left[\frac{p F}{V_{D}}\right]\left[\frac{e^{-\beta \tau}}{1-e^{-\beta \tau}}\right] D_{M}
$$

or

$$
C_{\mathrm{ss}}^{\min }=p\left[\frac{F}{V_{D}}\right]\left[\frac{e^{-\beta \tau}}{1-e^{-\beta \tau}}\right] D_{M}
$$


In these equations, $F$ is the fraction of each dose absorbed at steady state, $V_{D}$ is the volume of distribution, $D_{M}$ is the maintenance dose, $\beta$ is the apparent elimination rate constant, and $p$ is a proportionality constant and depends on the particular model. Often $\beta$ may be estimated from creatinine clearance measurements once a correlation has been established between $\beta$ and endogenous creatinine clearance. If equation 28 is used, then one correlates the values of $C_{s \mathrm{sin}}^{\mathrm{min}}$ with values of the product of $D_{M}$ and the part in the square bracket involving $\beta$. The proportionality constant or slope of the plot is the other part in the square bracket, namely $p F / V_{D}$. If equation 29 is used, then one must have knowledge of the value of $F$ and of $V_{D}$; for example, in the recent work of Jusko et al. (19) with digoxin. In this case, the proportionality constant is $p$.

If one is interested only in average steady-state blood levels, then equations 30-32 apply to linear systems and some nonlinear systems.

where

$$
\bar{C}_{\mathrm{ss}}=\frac{F D_{M}}{\dot{V}_{\mathrm{c} \tau} \tau}
$$

Hence

$$
\begin{gathered}
\bar{C}_{\mathrm{ss}}=\frac{\int_{t_{1}}^{t_{2}} C_{\mathrm{ss}}(t) d t}{\tau} \\
\tau=t_{2}-t_{1}
\end{gathered}
$$

where

$$
\frac{F}{\dot{\bar{V}}_{\mathrm{c} 1}}=\frac{\int_{0}^{\infty} C_{1}(t) d t}{D_{M}}=\frac{\int_{t_{1}}^{t_{2}} C_{\mathrm{ss}}(t) d t}{D_{M}}
$$

$$
\bar{V}_{\mathrm{c} 1}=\frac{(\text { dose })_{\text {i.v. }}}{\int_{0}^{\infty} C_{1}(t) d t}
$$

The equation of Wagner et al. (20) is written in model-independent fashion as equation 30 . Here, $\overline{\vec{V}}_{\mathrm{cl}}$ is the whole blood (plasma or serum) clearance and is defined by equation 33 . The other symbols have been defined before. One can obtain the ratio $F / \dot{V}_{\mathrm{cl}}$ from oral data, as indicated by equation 32 . Once an average value of this is known or a value for a particular patient, then predictions of $\bar{C}_{\mathrm{ss}}$ values for various values of $\tau$ and $D_{M}$ may be made using equation 30. Also, the estimate of $F / \bar{V}_{\mathrm{cl}}$ may be obtained from blood levels measured after a single dose of drug by making a ratio of the area from zero to infinite time to the dose.

\section{Amount of Drug in the Body}

The amount of drug remaining in the body at time $t$ after a bolus intravenous dose may be estimated by measurement of whole blood (plasma or serum) concentrations measured at times $t$, as shown in the following 
equations. The concentration-time data are fitted to a polyexponential equation as indicated by equation 34 :

$$
C=\sum_{i=1}^{n} I_{i} e^{-E_{i} t}
$$

The clearance is then calculated with equation 35 :

$$
\overline{\dot{V}}_{\mathrm{cl}}=\frac{D}{\int_{0}^{\infty} C d t}=\frac{D}{\sum_{i=1}^{n} I_{i} / E_{i}}
$$

The amount of drug remaining in the body at time $T\left(A_{B}\right)$ is then calculated with equation 36 .

$$
A_{B}=D-\overline{\bar{V}}_{\mathrm{cl}} \int_{0}^{T} C d t=D-\overline{\dot{V}}_{\mathrm{c} 1} \sum_{i=1}^{n} \frac{I_{i}}{E_{i}}\left(1-e^{-E_{i} T}\right)
$$

The volume of the central compartment, $V_{c}$, may be estimated with equation 37 , and the amount of drug in the central compartment, $A_{c}$, at time $T$ calculated with equation 38 :

$$
\begin{gathered}
V_{c}=\frac{D}{\sum_{i=1}^{n} I_{i}} \\
A_{c}=V_{c} \cdot C
\end{gathered}
$$

The amount of drug in compartments other than the central compartment at time $T, A_{0}$, may be calculated with equation 39 :

$$
A_{0}=A_{B}-A_{c}
$$

Analysis of data by hand or electronic calculator is often laborious and requires a prohibitive amount of time. Also, the "answers" obtained by digital computer are often better than the "answers" which may be obtained either by hand or by electronic calculator. Fortunately, the operations involved in pharmacokinetic analysis of data are generally systematic and therefore lend themselves to computer programming and solution by machine.

Sedman and Wagner have arranged to distribute the program AUTOAN (17) on a world-wide basis. AUTOAN is a Fortran IV digital computer program for automatic analysis of concentration-time data described by pharmacokinetic models involving first-order and/or Michaelis-Menten elimination kinetics. AUTOAN is used in conjunction with the 1969 version of the program NONLIN, distributed by Dr. Carl M. Metzler of the Upjohn Company in Kalamazoo, Michigan. The combination of AUTOAN and NONLIN provides for the complete pharmacokinetic analysis of whole 
blood (plasma or serum) concentration-time data. Only "raw" concentration-time data, and specification of parameters which indicate route of administration, weighting scheme desired, etc., are required for its use. AUTOAN analyzes concentration-time data as follows:

a. The presence or absence of Michaelis-Menten kinetics is determined.

b. If elimination kinetics are first order (as determined by the program), then the optimum polyexponential equation is fitted to the data; this may have from one to five exponential terms. The numerical values of the coefficients and the exponents of this optimum equation are printed out as well as similar values for lower-degree polyexponential equations which were determined in the process of reaching the optimum equation. The program then chooses an appropriate model for the data set from a library of models and computes initial estimates of the parameters of the model from the coefficients and exponents of the optimum polyexponential equation.

c. Final least-squares estimated of the model parameters are then obtained by NONLIN, performing a nonlinear least-squares fit of the data to the chosen model.

d. If elimination kinetics are those of Michaelis and Menten, the program will analyze the data if they obey the one-compartment open model with Michaelis-Menten elimination kinetics; if a more complicated disposition model is involved the program will so indicate, but does not provide a least squares fit. AUTOAN has been thoroughly tested and gives results comparable to or better than those obtained by laborious manual techniques. This program enables rapid, economical, and accurate computer analysis of pharmacokinetic data.

Some advantages of using AUTOAN are as follows:

a. Data are extended by interpolation of one data point between each pair of observed points using spline and Akima methods as reported by Fried and Zeitz (16). ${ }^{3}$ These interpolated points are used only during the derivation of the polyexponential equations and not during the final least-squares fitting of the data to the model chosen by the computer.

b. The program does all curve stripping automatically. The optimum polyexponential equation reported is, in general, a better one (i.e.,

\footnotetext{
${ }^{3} \mathrm{~A}$ second version of AUTOAN does not interpolate points, and also lag times have been introduced into the polyexponential equations. Arrangements for modifying the first version to include these additions may be made by contacting the author.
} 
lower sum of squared deviations) than that which is obtained using the back-projection technique and an electronic calculator.

c. The program uses a statistical criterion to distinguish first-order elimination from Michaelis-Menten elimination kinetics.

d. It automatically chooses one of a library of 17 models and outputs results of the nonlinear least-squares fit of the observed data to the chosen model.

e. The results printed out by AUTOAN may be used in model-independent equations, such as reported herein, or the parameters of the model chosen by the program may be used in equations for the particular model.

\section{NONLINEAR PHARMACOKINETICS}

My third thesis stated that when nonlinear pharmacokinetics apply it is necessary to obtain a partial or complete model to make accurate predictions. I will indicate some support for this thesis now.

DiSanto and Wagner (3) administered five different doses of methylene blue to a dog at different times by bolus intravenous injection and measured whole blood concentrations as a function of time after each of the doses. Each set of concentration-time data was very well fitted to the equations appropriate to the two-compartment open model with bolus intravenous injection. However, examination of the estimated parameters indicated that the parameters changed with dose. Hence one set of parameters was of no use in making predictions of whole blood concentrations for another dose of the drug. When the same data were fitted to the nonlinear tissue-binding model with one type of fluid and one type of tissue, then consistent values of the parameters were obtained. Also, it was possible to simultaneously fit all five sets of data to the model and estimate only one value of each of $A, B$, and $K$ from the data. Hence, in this case, the nonlinear model appeared to adequately describe the data and would allow prediction of blood levels following other doses within the same range, and, possibly, outside the range studied.

Historically, it has been assumed that ethyl alcohol is metabolized in man at a zero-order or constant rate and that the evidence for this is a pseudolinear phase in the blood concentration-time curve for some time starting after the peak alcohol concentration and continuing until there is marked curvature at the tail end of the curve on Cartesian coordinate graph paper. Wagner (21) clearly showed that if Michaelis-Menten elimination kinetics apply then the slope of the pseudolinear decline will increase with increase in dose ; hence the data cannot be explained by zero-order elimination. In the paper of Wagner (21), this conclusion was reached by a simula- 
tion technique. However, we now have human data resulting from administration of four different doses of ethanol to eight different subjects which give the same result. We have also data for seven normal volunteers in whom ethanol was infused at a constant rate over a 2 -hr period and the entire time course of capillary blood alcohol concentrations followed. The downslope data are fitted well to the one-compartment open model with Michaelis-Menten elimination, and application of a modified Wagner-Nelson method allowed back-calculation of the infusion rate with relatively small errors. However, even after publication of the paper of Wagner (21), the zero-order concept is still being repeated (22). There are still problems in interpreting some ethanol blood concentration data, as our future publications will indicate. However, it does appear that the Michaelis-Menten equation does explain the elimination phase adequately.

\section{REFERENCES}

1. L. Z. Benet. General treatment of linear mammillary models with elimination from any compartment as used in pharmacokinetics. J. Pharm. Sci. 61:536-541 (1972).

2. L. Michaelis and M. L. Menten. Die Kinetik der Invertinwirkung. Biochem. Z. 49:333-369

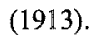

3. A. R. DiSanto and J. G. Wagner. Pharmacokinetics of highly ionized drugs. III. Methylene blue blood levels in the dog and tissue levels in the rat following intravenous injection. J. Pharm. Sci. 61:1090-1094 (1972). J. G. Wagner. Biopharmaceutics and Relevant Pharmocokinetics, Drug Intelligence Publications, 1st ed., Hamilton, Ill., 1971, pp. 302-317.

4. A. J. Sedman and J. G. Wagner. Quantitative pooling of Michaelis-Menten equations with parallel metabolite formation paths. J. Pharmacokin. Biopharm. 2:149-160 (1974).

5. K. B. Bischoff, R. L. Dedrick, D. S. Zaharko, and J. A. Longstreth. Methotrexate pharmacokinetics. J. Pharm. Sci. 60:1128-1133 (1971).

6. J. C. K. Loo and S. Riegelman. A new method for calculating intrinsic absorption rates of drugs. J. Pharm. Sci. 57:918-928 (1968).

7. J. G. Wagner. Application of the Loo-Riegelman absorption method. J. Pharmacokin. Biopharm. 3:51-67 (1975).

8. J. G. Wagner and E. Nelson. Percent absorbed time plots derived from blood level and/or urinary excretion data. J. Pharm. Sci. 52:610-611 (1963).

9. J. G. Wagner. Application for the Wagner-Nelson absorption method to the two compartment open model, J. Pharmacokin. Biopharm. 2:469-486 (1974).

10. K. S. Albert, A. J. Sedman, and J. G. Wagner. Pharmacokinetics of orally administered acetaminophen in man. J. Pharmacokin. Biopharm. 2:381-393 (1974).

11. M. Rowland. Influence of route of administration on drug bioavailability, J. Pharm. Sci. 67 : 70-74 (1972).

12. K. S. Albert, M. R. Hallmark, E. Sakmar, D. J. Weidler, and J. G. Wagner. Pharmacokinetics of diphenhydramine in man. J. Pharmacokin. Biopharm. 3:159-170 (1975).

13. J. G. Wagner. Method of estimating relative absorption of a drug in a series of clinical studies in which blood levels are measured after single and/or multiple doses. J. Pharm. Sci. 56:652-653 (1967).

14. A. Rescigno and G. Segre. Drug and Tracer Kinetics, Blaisdell, Waltham, Mass., 1966, p. 102.

15. L. Z. Benet and C.-W. N. Chiang. The use of deconvolution methods in pharmacokinetics. In Abstracts of Papers presented at the 13th National Meeting of the APhA Academy of Pharmaceutical Sciences, Chicago, November 5-9, 1972, Vol. 2, No. 2, pp. 169-171. 
16. J. Fried and S. Zeitz. Curve fitting by spline and Akima methods: Possibility of interpolation errors and its suppression. Phys. Biol. Med. 18:550-558 (1973).

17. AUTOAN, a Time-Sharing Digital Computer Program, available from Publication Distribution Service, 615 East University Avenue, Ann Arbor, Mich. 48106.

18. E. Krüger-Thiemer. Formal theory of drug dosage regimens. I. J. Theor. Biol. 13:212 (1966).

19. W. J. Jusko, S. J. Szefler, and A. L. Goldfarb. Pharmacokinetic design of digoxin dosage regimens in relation to renal function. J. Clin. Pharmacol. 14: 525-535 (1974).

20. J. G. Wagner, J. I. Northam, G. D. Alway, and O. S. Carpenter. Blood levels of drug at the equilibrium state after multiple dosing. Nature 207:1301-1302 (1965).

21. J. G. Wagner. Properties of the Michaelis-Menten equation and its integrated form which are useful in pharmacokinetics. J. Pharmacokin. Biopharm. 1:103-121 (1973).

22. C. A. M. Van Ginneken, J. M. Van Rossum, and H. L. J. M. Fleuren. Linear and nonlinear kinetics of drug elimination. I. Kinetics on the basis of a single capacity-limited pathway of elimination with and without simultaneous supply-limited elimination. J. Pharmacokin. Biopharm. 2:395-415 (1974). 